\title{
Effectiveness of Three Different Toothpaste Formulations in Reduction of Plaque and Gingivitis: A Comparative Clinical Study
}

\author{
${ }^{1}$ Amit Bhardwaj, ${ }^{2}$ Shalu V Bhardwaj \\ ${ }^{1}$ Senior Lecturer, Department of Periodontics, SGT Dental College, Gurgaon, Haryana, India \\ ${ }^{2}$ Dentist, Private Practitioner, Gurgaon, Haryana, India
}

Correspondence: Amit Bhardwaj, Senior Lecturer, Department of Periodontics, SGT Dental College, Gurgaon, Haryana, India e-mail: amitmds1980@ rediffmail.com

\begin{abstract}
The role of dental plaque in gingivitis and periodontitis is well established. It is commonly observed that daily brushing often fails to achieve and maintain optimum gingival health. In order to improve the efficacy of the self-performed mechanical tooth cleaning procedure, antimicrobial substances like chlorhexidine, metallic ions, fluorides and various phenolic compounds have been evaluated, as such adjuvants in clinical trials that have been added to dentifrice used during tooth brushing. Triclosan $(2,4,4$ ' trichloro-2'hydroxyphenylether) is a nonionic antibacterial agent with broad spectrum activity against both gram-positive and gram-negative organisms. Recently, triclosan was introduced in toothpaste and mouth rinses for control of plaque and gingivitis. However, maximum antiplaque activity is shown when triclosan is used in combination with agents like copolymer polyvinyl methyl ether maleic acid (PVM/MA). The PVM/MA copolymer retains the triclosan on the soft and hard surfaces of the oral cavity for several hours. This study aims in proving efficacy of triclosan copolymer toothpaste over the triclosan alone and non-triclosan toothpaste in reduction of plaque and gingivitis.

Keywords: Triclosan, Gingivitis, Toothpaste.
\end{abstract}

\section{INTRODUCTION}

Findings from different areas of research have documented the importance of supra- and subgingival plaque removal in the treatment of gingivitis and periodontitis. ${ }^{1-3} T$ he need of careful plaque control and, at inflamed sites, repeated subgingival debridement in the maintenance phase after active periodontal therapy was al so demonstrated. ${ }^{4-6}$ Smulow et al ${ }^{7}$ observed that supragingival plaque control, delivered by professional, had a pronounced influence on the qual ity and quantity of subgingival microbiota. Similar findings were presented by $\mathrm{MCN}$ abb ${ }^{8}$ and Hellstrom et al. ${ }^{9}$

This study attempts to compare the reduction of plaque and gingivitis among three toothpaste formulations containing mainly of:

1. Triclosan and copolymer fluoridated toothpaste

2. Triclosan and fluoridated toothpaste

3. Fluoridated non-triclosan toothpaste.

\section{OBJECTIVE OF THE STUDY}

This study attempts to compare the reduction of plaque and gingivitis among three toothpaste formulations:

1. Triclosan and copolymer flouridated toothpaste (TCD) (test group)

2. Triclosan and flouridated toothpaste (TC) (test group)

3. Flouridated non-triclosan toothpaste (TFD) (control group).

\section{METHODS OF COLLECTION OF DATA}

\section{Inclusion Criteria}

Healthy individuals between 18 and 35 years of age using nonfluoridated and non-triclosan containing toothpastes with minimal staining of teeth were selected. The individuals must be having at least 24 intact natural teeth, free from active caries, overt periodontitis or large restorations in gingival areas, and absence of oral infection and sensitive teeth.

\section{Exclusion Criteria}

Individuals wearing denture or those with crowns and bridges, those showing probing depth more than $3 \mathrm{~mm}$ or on long-term antibiotic or other drug therapy were excluded from study. Expecting and nursing mothers were al so excluded.

\section{Study Design}

Total 30 subjects were taken for the study. For each group 10 subjects were randomly selected. Baseline val ues for gingival index (L oe and silness), gingival bleeding index, and Turesky modification for plaque was assessed.

Toothpastes and medium bristles toothbrush were distributed and subjects were instructed to brush their teeth in their usual and customary manner for 3 minutes twice daily. Clinical assessment was done on day 0 and at day 21 . For all experimental groups, plaque index (PI), gingival bleeding index (GBI) and gingival index (GI) were recorded. 


\section{STATISTICAL ANALYSIS}

ANOVA for testing main score of gingival index, gingival bleeding index, plaque index and comparing the post effects of three toothpaste formulations together. Pair T-test used for evaluating intragroup changes from baseline.

Percentage distribution of plaque index, gingival index, gingival bleeding index for all toothpaste formulations was al so done.

\section{RESULTS}

\section{Plaque and Gingivitis at Day 0}

A t day 0 , in all the data collected, most of study subjects were showing quite similar scores of GI, PI, GBI for all groups, that means they were well balanced.

\section{Plaque and Gingivitis at Day 21}

Results have shown that among the various toothpaste formulations, triclosan containing formulation was effective in removing plaque. Statistical analysis showed that triclosan copolymer containing toothpaste was even better than triclosan containing toothpaste.

However, there was no statistical difference between the two toothpaste formulations in terms of plaque removal.

\section{Gingivitis and Bleeding at 21 Days}

Statistical analysis showed differences among the treatments related to gingivitis and bleeding indices. The only formulation containing triclosan and PVM/MA was able to reduce significantly gingivitis development and bleeding in comparison with the control.

There was significant differences in reduction of gingival bleeding score seen after the use of triclosan copolymer when compared with only triclosan containing toothpaste and nontriclosan fluoridated toothpaste.

Triclosan copolymer containing toothpaste showed more 0, 1, 2 and less 3, 4, 5 scores for plaque index (Fig. 1).

Triclosan copolymer also showed more 0, 1 and less 2, 3 score of gingival index, thus showing significant reduction in gingivitis (Fig. 2).

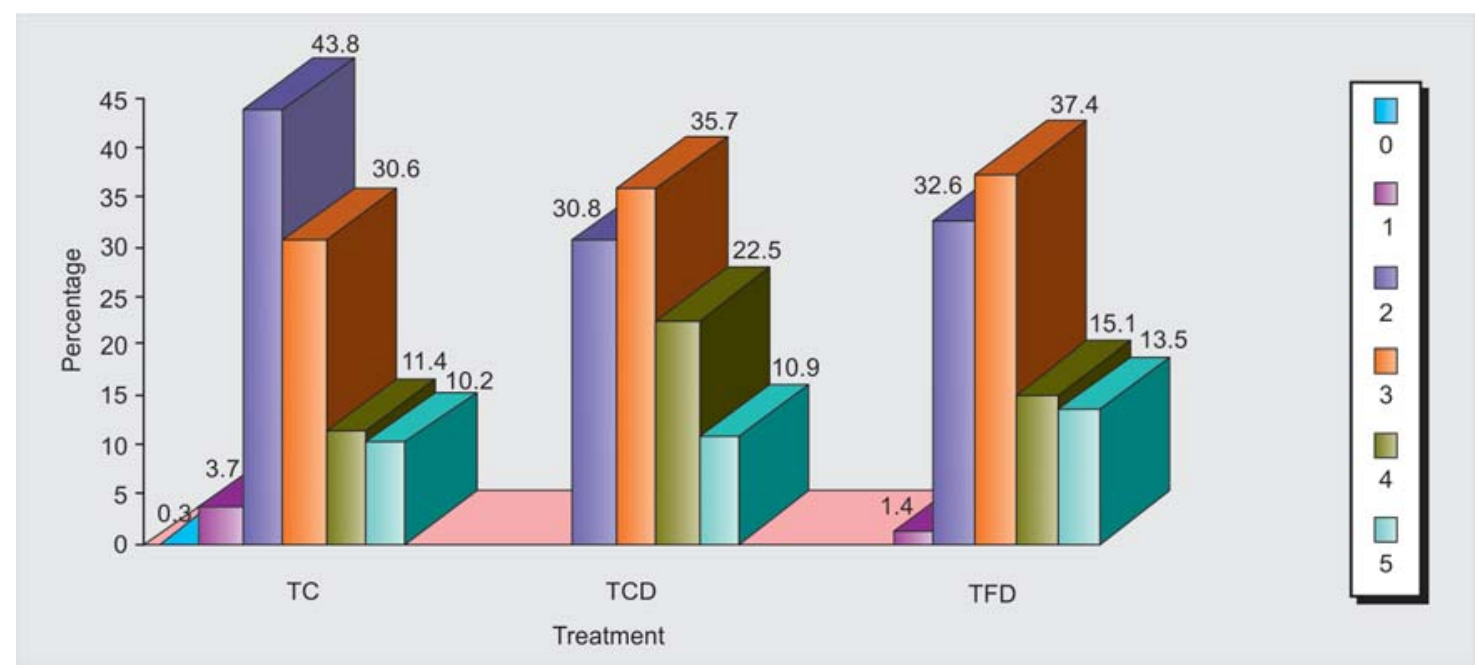

Fig. 1: Percentage distribution of plaque index (PI)

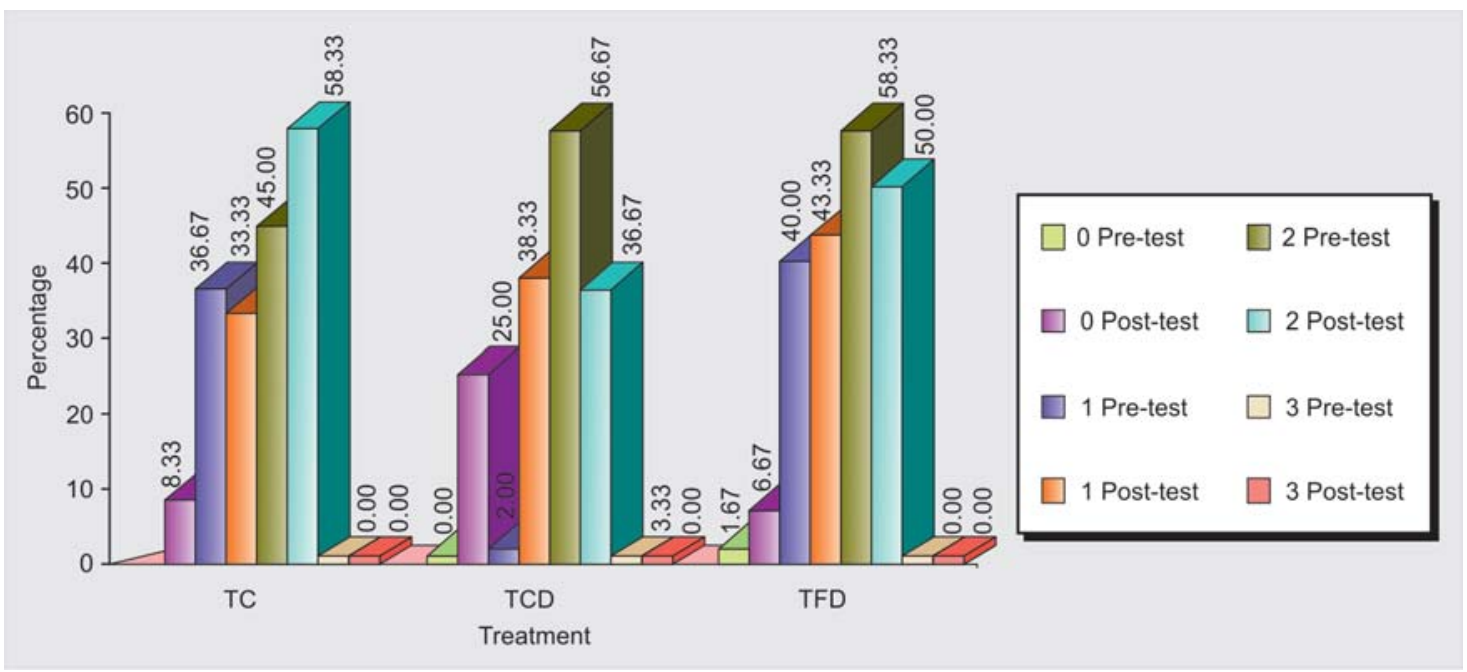

Fig. 2: Percentage distribution of gingival index (GI) 


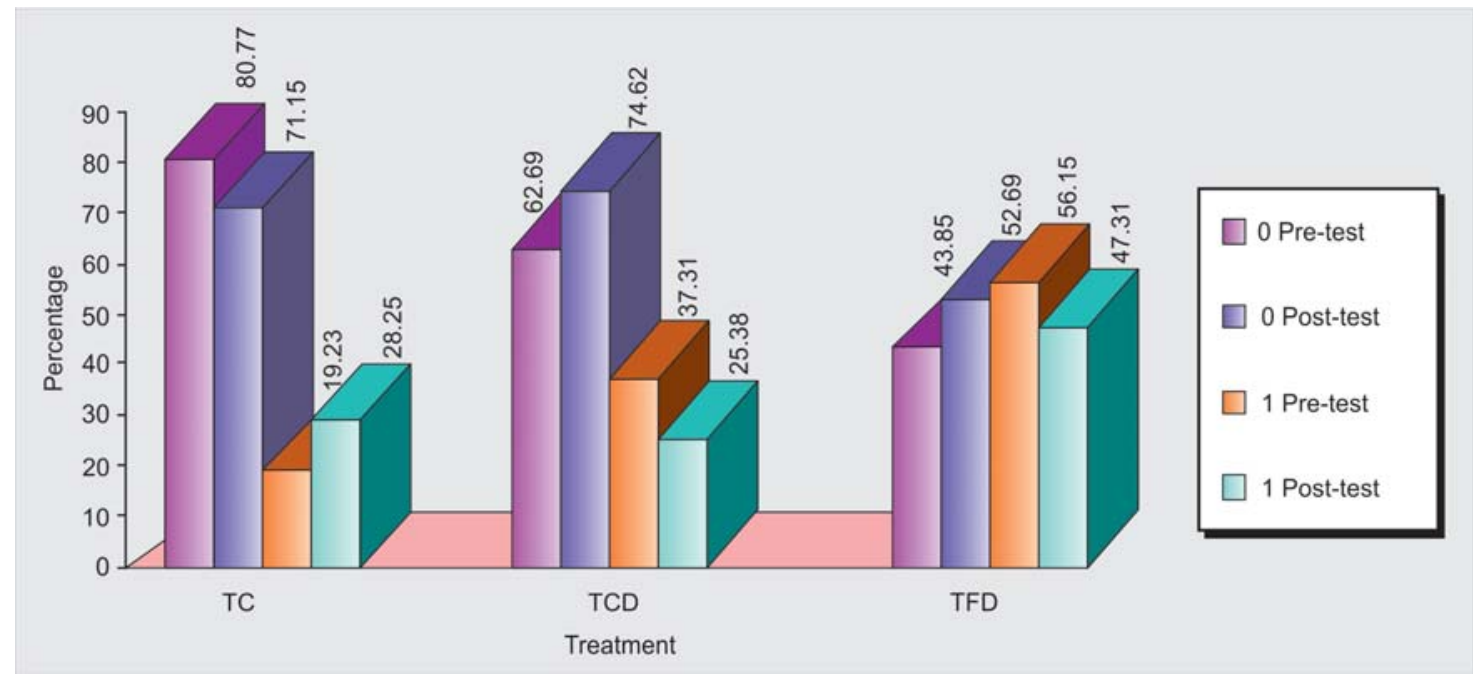

Fig. 3: Percentage distribution of gingival bleeding index (GBI)

B leeding index also showed more of zero score as compared to one score in relation to use of triclosan containing toothpaste (Fig. 3).

Hence, results showed that among various toothpaste formulations triclosan copolymer containing formulation was effective in removing plaque when compared with fluoridated non-triclosan and fluoridated triclosan containing toothpaste. The only formulation containing triclosan and PVM M M A was able to reduce significantly gingivitis development and bleeding in comparison with the test and control group.

\section{DISCUSSION}

Lindhe ${ }^{10}$ and Palamo et al ${ }^{11}$ showed efficiency of dentifrices containing triclosan and copolymer or triclosan alone when compared to control, in simple solutions, at relatively high concentrations ( $0.2 \%$ ) and dose ( $20 \mathrm{mg}$ twice per day) retention of about 3 hours on oral mucosa and 8 hours in dental plaque after use. Triclosan has moderate plaque inhibitory action and antimicrobial substantivity. ${ }^{12}$ The activity of triclosan appears to be enhanced by the addition of zinc citrate and copolymers polyvinylmethyl ether maleic acid. ${ }^{13,14}$ Copolymers are important for enhancement of the antiplaque effect of triclosan as it increases the retention of triclosan. It has antibacterial as well as antifungal and antiviral properties. In addition, because of its nonionic character, triclosan is compatible with other desirable ingredients in dentifrices. ${ }^{15}$ The mechanisms of its antiseptic action is by acting on microbial cytoplasmic membrane, including leakage of cellular constituents and thereby causing lysis of microorganisms. Evidence has accumulated to suggest that triclosan in itself does not produce optimal plaque inhibitory effects without addition of other chemical which increases its antibacterial effect. One approach is to add PVM/MA copolymer. Dentifrice containing $0.3 \%$ triclosan, 2.0\% PV M /M A copolymer, $0.243 \%$ sodium fluoride in a $17 \%$ dual silica base is efficacious for the control of established supragingival plaque and gingivitis. ${ }^{16}$ Triclosan copolymer containing toothpaste has been demonstrated to be highly effective in plaque and gingivitis control. ${ }^{17}$

\section{CONCLUSION}

Evidence has accumulated to suggest that triclosan in itself does not produce optimal plaque inhibitory effects without addition of other chemicals which increase its antibacterial effect. One approach is to add PV M /M A copolymer. Triclosan PV M /M A experimental dentifrice was more effective in plaque, gingivitis and gingival bleeding reduction than with other dentifrices, and such differences may be explained by the antimicrobial synergism of the active ingredients of the formulation.

\section{REFERENCES}

1. Cobb CM. Proceedings of the 1996 world workshop in periodontics: N onsurgical pocket therapy. Mechanical A nnals of Periodontology 1996;1:443-90.

2. Kieser. N onsurgical periodontal therapy: Proceedings of the 1st European workshop on periodontology. L ondon: Quintessence 1994:131-58.

3. Wachtel. Surgical periodontal therapy: Proceedings of the 1st European workshop on periodontology. L ondon: Quintessence 1994;159-71.

4. Axellson, Lindhe. Effect of controlled oral hygiene procedures on caries and periodontal disease in adults. J ournal of Clinical Periodontology 1981;8:239-48.

5. B ostanci, A rpak. L ong-term evaluation of surgical periodontal treatment with and without maintenance care. J ournal of $\mathrm{N}$ ihon University School of Dentistry 1991;33:152-59.

6. Wilson, et al. Tooth loss in maintenance patients in a private periodontal practice. Journal Of Periodontology 1987;58: 231-35.

7. Smulow, et al. The effect of supragingival plaque removal on anaerobic bacteria in deep periodontal pockets. Journal of A merican dental association 1983;107:737-42.

8. $M c N a b b$, et al. Supragingival cleaning three times a week: The microbiological effects in moderately deep pockets. J ournal of Clinical Periodontology 1992;19:348-56. 
9. Hellstorm, et al. The effect of supragingival plaque control on the subgingival microflora in human periodontitis. Journal of Periodontology 1996;23:934-40.

10. Lindhe J, R osling B, Socransky SS, V olpe AR. The effect of a triclosan-containing dentrifice on established plaque and gingivitis. Journal of Clinical Periodontology 1993;20:327-34.

11. Palamo, et al. The effect of three commercially available dentifrices containing triclosan on supragingival plaque formation and gingivitis: A six month clinical study. International Dental J ournal 1995;44:75-81.

12. J enkins, et al. Triclosan and sodium lauryl sulfate mouth rinses. Effects on 4 days plaque regrowth. J ournal of Periodontology 1991;18:145-48.

13. Cummins. M echanisms of actions of clinically proven antiplaque agents. Clinical and biological aspects of dentifrices. Oxford: oxford university press 1992;205-28.
14. Gaffar A, Scherl D, A fflitto J, Coleman EJ. The effect of triclosan on mediators of gingival inflammation. Journal of Clinical Periodontology 1995;22:480-84.

15. V an der Ouderaa FJ, et al. Delivery systems for agents in supra and sub gingival plaque control. Journal of Dental Research 1989;68:1617-24.

16. M ateu FA, Boneta AE, DeV izio W, Stewart B, Proskin HM. A clinical investigation of the efficacy of two dentifrices for controlling established supragingival plaque and gingivitis. J Clin Dent 2008;19(3):85-94.

17. Vered $Y$, Zini A, M ann J, DeV izio W, Stewart B, Zhang Y P, Garcia $L$. Comparison of a dentifrice containing $0.243 \%$ sodium fluoride, $0.3 \%$ triclosan, and $2.0 \%$ copolymer in a silica base, and a dentifrice containing $0.243 \%$ sodium fluoride in a silica base: A three-year clinical trial of root caries and dental crowns among adults. J Clin D ent 2009;20(2):62-65. 